\title{
Antioxidant Capacity and Consumer Acceptability of Spiced Black Tea
}

\author{
Simon Oduor Ochanda ${ }^{1}$, John Kanyiri Wanyoko ${ }^{1} \&$ Henrik Kipngeno Ruto ${ }^{1}$ \\ ${ }^{1}$ Kenya Agricultural and Livestock Research Organization - Tea Research Institute (KALRO -TRI), Kenya \\ Correspondence: Henrik Kipngeno Ruto, Kenya Agricultural and Livestock Research Organization - Tea \\ Research Institute (KALRO -TRI), Kenya. E-mail: rutohenrik@yahoo.com
}

Received: August 4, 2015 Accepted: October 14, 2015 Online Published: November 16, 2015

doi:10.5539/jfr.v4n6p104

URL: http://dx.doi.org/10.5539/jfr.v4n6p104

\begin{abstract}
Value addition of bulk curl tear cut (CTC) black tea is important to meet emerging customer needs and address challenges in a competitive beverage market. Spicing of the tea is one way of value addition but little or no research has been done on the biochemical effect of blending tea with spices and consumer acceptability. A study was conducted to determine the effect of spices on consumer acceptability; pricing and anti-oxidant capacities of black CTC tea consumed in the Kenyan market. Six spices and a spice mix including; ginger, lemon grass, nutmeg, cinnamon, rosemary and tea masala were used to develop aerated spice tea. The spice mix-tea masala comprised of ginger, cinnamon, cardamoms, cloves, black pepper and nutmeg. The threshold levels of spice-tea blends for commercial purposes were demonstrated using three highly rated spices i.e. cinnamon, lemon grass and ginger. Economic costing was done using the cinnamon spiced-tea. The results showed that black tea had the highest antioxidant activity of $92.66 \%$ against that of the highest spice cinnamon $89.89 \%$. Antioxidant activity of spiced tea significantly $(\mathrm{P} \leq 0.05)$ decreased with the quantity of added spice. Spices significantly $(\mathrm{P} \leq 0.05)$ increased consumer preference of the black tea and the preferred spice-mix ratios also differed. Some spices were preferred more than others as shown by the three best rated spice-tea mixes including; cinnamon at $10 \%$ lemon grass at $5 \%$ and ginger at $15 \%$ which had mean scores of $6.74,6.35$ and 6.58 respectively on a hedonic scale.
\end{abstract}

Keywords: consumer acceptability, spiced tea, sensory evaluation, antioxidant activity, value addition

\section{Introduction}

Kenya consumes 5\% of the tea it produces as black (aerated) Cut Tear and Curl Tea (Anon., 2011; ITC, 2010). A recent survey conducted by Tea Research Institute, showed over 50\% of imported teas sold in retail stores and supermarkets are spiced and that a growing number of packers and blenders were increasingly developing flavored teas.

Currently the world tea market is facing competition from other beverages and there are an increasingly high number of consumers with different needs. Value addition is one way of meeting such challenges. According to (Herath \& De Silva, 2011) value addition of tea can be done through brand building and product differentiation. Spicing of tea is one way of product differentiation and brand development. Spices have been widely used to modify the flavors of foods and beverages so as to make them more appealing to consumers. Different spices are suitable for different foods while others have a broad range of application. In the Kenyan market, some spices are used with a variety of foods while others are specifically sold for specific foods like tea (tea masala lemongrass and ginger) and rice (pilau masala).

Apart from the flavors and aroma, spices have some nutritional value, exhibit antioxidant activity and antimicrobial effect among other numerous health benefits which can be championed to sell the tea product (Benkeblia, 2004; Bullerman, Lieu, \& Seier, 1977; Choi et al., 2002; Holt, Katz, \& Kirshoff, 2005; Jagetia, Baliga, Venkatesh, \& Ulloor, 2003; Lelgo, Kamunya, Ochanda, \& Wanyoko, 2011; Ochanda et al., 2011; Ruto, Moseti, Koech, \& Wanyoko, 2014). This study was focused on evaluating the effects of adding spices to black tea manufactured from cultivar TRFK 6/8 against consumer acceptability and effect on antioxidant potential of blended spiced tea. 


\section{Materials and Method}

The work was carried out in phases which included; processing of the aerated black tea, developing flavored teas by blending the processed black tea with selected spices including ginger, lemon grass, nutmeg, cinnamon, tea masala, and rosemary and determining consumer preferences of the products. Different ratios of tea and spices were blended and the resulting products assessed using a sensory panel. The results of the sensory panel were statistically analyzed to identify consumer preferences.

\subsection{Raw Materials}

Powdered spices manufactured by tropical heat, including ginger, lemon grass, nutmeg, cinnamon, tea masala, and rosemary were obtained from local retail stores in Kericho, Kenya. Green leaf from TRFK 6/8 variety was used. This clone is a standard for quality black tea (Karori, Wachira, Ngure, \& Mireji, 2014; Ochanda, Wanyoko, \& Ruto, 2015).

\subsection{Black Tea Processing}

Black and green tea from cultivar TRFK 6/8 was processed in a miniature factory at Tea Research Institute (TRI), Kericho, Kenya and used to develop the flavored teas. Fresh leaf was plucked and processed using the method described by (S O Ochanda et al., 2011; Willson \& Clifford, 1992). The first step after plucking was withering and was done by laying out fresh leaves on a withering trough for $18 \mathrm{hrs}$ to achieve a moisture content of $65 \%$. The withered leaf was then macerated in a CTC machine to get a dhool. The dhool was put in a fermentation chamber for 45-90 minutes to achieve a copper red colour and ensure optimum oxidation with theaflavins to thearubigins ratio of 1:10. Drying was then performed in a fluid bed dryer at temperatures of $100^{\circ} \mathrm{c}$ to achieve a moisture content of $3-4 \%$. The tea at this point is black and has established an aroma. The resulting tea was subjected to biochemical assay to profile its composition before blending with spices.

\subsection{Spiced Aerated Tea Development}

The research was carried out in three phases; (1) Processing and development of aerated (black) spiced tea products by blending the aerated tea with the spices at different ratios, (2) Determination of the effects of the additives on antioxidant capacities and consumer acceptability of the teas and (3) Economic, statistical analysis and data interpretation. Initially the six spices were blended with the tea at high percentages of 25,50 and $75 \%$ and later lowered to 5, 10, 15 and $25 \%$ to determine economic threshold levels.

\subsection{Biochemical Assays}

\subsubsection{Determination of Catechins and Polyphenols}

Aerated (black) tea from TRFK 6/8 was assayed for quality through catechins, caffeine and Gallic acid profiling. Extraction of catechins and polyphenols was done according to the procedure described by Karori et al (Karori, Wachira, Wanyoko, \& Ngure, 2007). HPLC analysis was done according to ISO procedure and as described by (ISO14502-2-2005E., 2005; Karori et al., 2007). Catechin identification was done by comparing the retention times of samples and standards under similar conditions. Total polyphenol content was determined using the folin-ciocalteu method as described by (Kerio, Wachira, Wanyoko, \& Rotich, 2013; Koech \& Wachira, 2013)

\subsubsection{Determination of Plain Tea Quality Parameters}

Oxidation products i.e. theaflavins and thearubigins, are responsible for black tea quality and can be determined by a method described by ref (Mohd Nasir, 2012; Roberts \& Smith, 1961). This method was also used to determine other quality parameters such as color and brightness, indicators of tea quality.

\subsubsection{Determination of Antioxidant Properties}

The antioxidant properties of all the spices and spiced aerated tea from TRFK 6/8 were determined using the method described by (S O Ochanda et al., 2011). The stable 2,2-diphenyl-1-picrylhydrazyl radical (DPPH) analar grade, was used for determination of free radical scavenging ability of aerated tea and spice aerated tea.

\subsection{Sensory Evaluation}

Thirty six (36) developed spiced black tea products from TRFK 6/8, were subjected to sensory evaluation. Two (2) grams of each product were infused in $250 \mathrm{mls}$ of with hot water and sweetened with 2grams of sugar. The brew was served hot in clear $175 \mathrm{ml}$ glasses. Thirty (30) panelists were used to evaluate all the products. To avoid olfactory fatigue, each spiced product was evaluated at different sessions. Panelists used were of both male and female and their ages ranged between 18-65yrs (Meilgaard, Civille, \& Carr, 1999; Miller \& Bartoshuk, 1991; Moraes, 1993). Explation of the test to the panelists was done to ensure that they understood what the exercise entailed. Sensory attributes and the questionnaire were explained (IDF, 1987; Meilgaard et al., 1999; Miller \& 
Bartoshuk, 1991; Moraes, 1993). Sensory panelists' used clean water to rinse palate after every evaluation. Sensory evaluation was done in a room with ambient light. Five attributes were evaluated; taste, smell, texture, acceptability, and color, using a 9 point hedonic scale, 1=dislike extremely; $2=$ dislike much; $3=$ dislike moderately; $4=$ dislike slightly, $5=$ neither dislike nor like, $6=$ like slightly; $7=$ like moderately; $8=$ like much, 9=like extremely (IDF, 1987; Meilgaard et al., 1999). Taste as used in this article was a consumer assessment of flavor. The sensory evaluation data was presented as means of five groups of panelist's scores using SAS 9.1 Statistical package (SAS Institute Inc., n.d.). Significance was evaluated at P $\leq 0.05$ (Carpenter, 1960; Cass, 1980; Cochran, 1977).

\subsection{Economic Evaluation}

The best rated spiced tea was used to determine economic threshold values for the consumer market. Prices of aerated (black) tea products, cinnamon, tea masala, ginger (Tangawizi) spices and their tea-mixes were obtained and used for economic evaluation and analysis. Statistical evaluation was used to determine significant differences in prices $(\mathrm{P} \leq 0.05)$ (Carpenter, 1960; Cass, 1980; Cochran, 1977).

\section{Results and Discussion}

Spices significantly $(\mathrm{P} \leq 0.05)$ lowered antioxidant activities of the black tea from clone TRFK $6 / 8$ as shown in Table 1 below. As the spice to tea ratio was increased antioxidant activity was further lowered (Choi et al., 2002; Karori et al., 2007; Yen \& Duh, 1994; Zong-Mao, Wang, Xiao-Qingyou, \& Xu, 2005). This decline however, should not be used to rate the potential health benefits of spiced tea since have other intrinsic benefits of their own (Holt et al., 2005; Jagetia et al., 2003; Lai \& Roy, 2004; Nielsen \& Rios, 2000), but further studies should be done to evaluate health benefits of spiced black tea blends. Most of these spices used in the study have been associated with unlimited health benefits which may be useful during marketing of spiced tea (Benkeblia, 2004; Bullerman et al., 1977; Crespy \& Williamson, 2004; Crozier, Jaganath, \& Clifford, 2009; Guynot et al., 2003; Wang, Provan, \& Helliwell, 2000; Zong-Mao et al., 2005).

Table 1. Antioxidant capacities of selected Spices and TRFK 6/8

\begin{tabular}{llll}
\hline Spice & Spice (\%) & Tea (\%) & Antioxidant Activity (\%) \\
\hline N/A & N/A & 100 & $92.66^{\mathrm{a}} \pm 0.47$ \\
Cinnamon & 100 & N/A & $89.89^{\text {ef }} \pm 0.81$ \\
Ginger & 100 & N/A & $69.23^{\mathrm{k}} \pm 0.45$ \\
Rosemary & 100 & N/A & $89.47^{\mathrm{f}} \pm 0.50$ \\
Tea masala & 100 & N/A & $55.70^{\mathrm{l}} \pm 1.16$ \\
Nutmeg & 100 & N/A & $46.99^{\mathrm{m}} \pm 0.29$ \\
Ginger & 5 & 95 & $88.31^{\mathrm{g}} \pm 0.29$ \\
Ginger & 10 & 90 & $86.39^{\mathrm{h}} \pm 0.37$ \\
Ginger & 15 & 85 & $85.65^{\mathrm{i}} \pm 0.22$ \\
Ginger & 25 & 75 & $84.40^{\mathrm{i}} \pm 0.14$ \\
Ginger & 100 & N/A & $69.23^{\mathrm{k}} \pm 0.45$ \\
Cinnamon & 5 & 95 & $91.94^{\mathrm{b}} \pm 0.06$ \\
Cinnamon & 10 & 90 & $91.74^{\mathrm{b}} \pm 0.09$ \\
Cinnamon & 15 & 85 & $90.61^{\mathrm{cd}} \pm 0.04$ \\
Cinnamon & 25 & 75 & $89.51^{\mathrm{f}} \pm 0.47$ \\
Cinnamon & 100 & N/A & $89.89^{\mathrm{ef}} \pm 0.81$ \\
Nutmeg & 5 & 95 & $90.88^{\mathrm{c}} \pm 0.06$ \\
Nutmeg & 10 & 90 & $90.66^{\mathrm{cd}} \pm 0.04$ \\
Nutmeg & 15 & 85 & $90.51^{\mathrm{cde}^{\mathrm{de}} \pm 0.08}$ \\
Nutmeg & 25 & 75 & $90.04^{\mathrm{def}} \pm 0.04$ \\
Nutmeg & 100 & N/A & $46.99^{\mathrm{m}} \pm 0.29$ \\
Mean & & & $\mathbf{8 4 . 1 4}$ \\
LSD(0.05) & & & $\mathbf{0 . 7 0}$ \\
CV (\%) & & & $\mathbf{0 . 5 0}$ \\
\hline
\end{tabular}

Means in the same column with the same letters are not significantly different. $(P>0.5) . N / A=$ Not Added. 
Table 2(a). Effect of spices on consumer acceptability of black tea from TRFK 6/8

\begin{tabular}{|c|c|c|c|c|c|c|c|}
\hline Concentration & Spice & Taste & Smell & Texture & Acceptability & Color & Mean \\
\hline $0 \%$ & Ginger & $6.31^{\mathrm{a}} \pm 0.77$ & $5.94^{\mathrm{b}} \pm 0.92$ & $6.04^{\mathrm{a}} \pm 2.38$ & $5.78^{\mathrm{a}} \pm 2.10$ & $5.87^{\mathrm{a}} \pm 1.35$ & 5.88 \\
\hline $25 \%$ & & $6.24^{\mathrm{a}} \pm 0.32$ & $6.25^{\mathrm{ab}} \pm 1.01$ & $6.73^{\mathrm{a}} \pm 0.28$ & $6.54^{\mathrm{a}} \pm 0.71$ & $6.28^{\mathrm{a}} \pm 1.10$ & 6.24 \\
\hline $50 \%$ & & $6.75^{\mathrm{a}} \pm 0.54$ & $6.00^{\mathrm{a}} \pm 0.28$ & $6.30^{\mathrm{a}} \pm 1.26$ & $5.61^{\mathrm{a}} \pm 1.50$ & $5.77^{\mathrm{a}} \pm 1.04$ & 6.67 \\
\hline $75 \%$ & & $6.96^{\mathrm{a}} \pm 0.28$ & $6.53^{\mathrm{a}} \pm 1.22$ & $6.21^{\mathrm{a}} \pm 0.89$ & $6.61^{\mathrm{a}} \pm 1.30$ & $5.65^{\mathrm{a}} \pm 1.71$ & 6.35 \\
\hline Mean & & 6.57 & 6.18 & 6.32 & 6.13 & 6.17 & 6.29 \\
\hline $\operatorname{LSD}(0.05)$ & & 1.14 & 0.43 & 2.27 & 1.38 & 0.04 & \\
\hline$C V(\%)$ & & 8.68 & 3.45 & 17.99 & 11.29 & 0.33 & \\
\hline $0 \%$ & Lemongrass & $6.48^{\mathrm{b}} \pm 0.44$ & $6.67^{b} \pm 0.36$ & $6.52^{\mathrm{ab}} \pm 0.21$ & $6.81^{\mathrm{ab}} \pm 0.21$ & $6.57^{\mathrm{a}} \pm 0.80$ & 6.61 \\
\hline $25 \%$ & & $6.81^{\mathrm{b}} \pm 0.17$ & $6.53^{\mathrm{b}} \pm 0.30$ & $5.71^{\mathrm{b}} \pm 0.76$ & $6.23^{\mathrm{b}} \pm 0.65$ & $5.81^{\mathrm{a}} \pm 0.57$ & 6.22 \\
\hline $50 \%$ & & $7.29^{\mathrm{ab}} \pm 0.29$ & $7.19^{\mathrm{ab}} \pm 0.78$ & $6.76^{\mathrm{ab}} \pm 0.79$ & $7.14^{\mathrm{a}} \pm 0.29$ & $6.67^{\mathrm{a}} \pm 0.65$ & 7.01 \\
\hline $75 \%$ & & $8.10^{\mathrm{a}} \pm 0.59$ & $8.05^{\mathrm{a}} \pm 0.71$ & $7.52^{\mathrm{a}} \pm 0.44$ & $7.10^{\mathrm{a}} \pm 0.68$ & $6.62^{\mathrm{a}} \pm 0.58$ & 7.48 \\
\hline Mean & & 7.17 & 7.11 & 6.63 & 6.82 & 6.42 & 6.83 \\
\hline $\operatorname{LSD}(0.05)$ & & 0.84 & 1.08 & 1.06 & 0.63 & 1.4 & \\
\hline$C V(\%)$ & & 5.83 & 7.58 & 8.02 & 4.6 & 10.95 & \\
\hline $0 \%$ & Cinnamon & $4.86^{\mathrm{c}} \pm 0.46$ & $5.46^{\mathrm{a}} \pm 0.45$ & $5.82^{\mathrm{b}} \pm 0.17$ & $4.95^{\mathrm{b}} \pm 0.59$ & $6.48^{\mathrm{a}} \pm 0.50$ & 5.51 \\
\hline $25 \%$ & & $5.73^{b \mathrm{bc}} \pm 0.28$ & $6.27^{\mathrm{ab}} \pm 0.17$ & $6.56^{\mathrm{ab}} \pm 0.48$ & $5.96^{\mathrm{a}} \pm 0.07$ & $6.67^{\mathrm{a}} \pm 0.68$ & 6.24 \\
\hline $50 \%$ & & $6.69^{\mathrm{ab}} \pm 0.23$ & $6.52^{\mathrm{ab}} \pm 0.45$ & $6.37^{\mathrm{ab}} \pm 0.67$ & $6.50^{\mathrm{a}} \pm 0.11$ & $5.98^{\mathrm{a}} \pm 0.60$ & 6.41 \\
\hline $75 \%$ & & $7.74^{\mathrm{a}} \pm 0.75$ & $6.93^{\mathrm{a}} \pm 0.71$ & $6.95^{\mathrm{a}} \pm 0.35$ & $6.76^{\mathrm{a}} \pm 0.62$ & $5.86^{\mathrm{a}} \pm 0.75$ & 6.85 \\
\hline Mean & & 6.25 & 6.3 & 6.43 & 6.05 & 6.25 & 6.26 \\
\hline$L S D(0.05)$ & & 1.06 & 1.08 & 1.04 & 0.9 & 1.28 & \\
\hline$C V(\%)$ & & 8.45 & 8.61 & 8.1 & 7.44 & 10.22 & \\
\hline $0 \%$ & Nutmeg & $6.40^{\mathrm{a}} \pm 0.46$ & $6.69^{\mathrm{a}} \pm 0.99$ & $5.99^{\mathrm{a}} \pm 0.87$ & $5.33^{\mathrm{a}} \pm 0.97$ & $5.77^{\mathrm{a}} \pm 1.12$ & 6.04 \\
\hline $25 \%$ & & $5.99^{\mathrm{a}} \pm 1.49$ & $6.20^{\mathrm{a}} \pm 0.93$ & $5.94^{\mathrm{a}} \pm 0.37$ & $6.13^{\mathrm{a}} \pm 0.65$ & $6.32^{\mathrm{a}} \pm 0.88$ & 6.12 \\
\hline $50 \%$ & & $6.15^{\mathrm{a}} \pm 1.18$ & $5.88^{\mathrm{a}} \pm 0.97$ & $5.93^{\mathrm{a}} \pm 0.65$ & $5.44^{\mathrm{a}} \pm 1.07$ & $5.91^{\mathrm{a}} \pm 0.82$ & 5.86 \\
\hline $75 \%$ & & $5.22^{\mathrm{a}} \pm 0.83$ & $5.96^{\mathrm{a}} \pm 0.67$ & $5.60^{\mathrm{a}} \pm 0.49$ & $5.38^{\mathrm{a}} \pm 0.93$ & $6.03^{\mathrm{a}} \pm 0.36$ & 5.64 \\
\hline Mean & & 5.94 & 6.18 & 5.87 & 5.57 & 6.01 & 5.91 \\
\hline $\operatorname{LSD}(0.05)$ & & 1.24 & 0.81 & 1.32 & 1.08 & 1.19 & \\
\hline$C V(\%)$ & & 10.42 & 6.57 & 11.23 & 9.68 & 9.99 & B \\
\hline $0 \%$ & Rosemary & $6.02^{\mathrm{a}} \pm 0.77$ & $5.76^{\mathrm{a}} \pm 0.66$ & $6.02^{\mathrm{a}} \pm 1.13$ & $5.71^{\mathrm{a}} \pm 1.65$ & $6.34^{\mathrm{ab}} \pm 1.20$ & 5.97 \\
\hline $25 \%$ & & $6.28^{\mathrm{a}} \pm 0.31$ & $6.15^{\mathrm{a}} \pm 0.96$ & $6.14^{\mathrm{a}} \pm 0.48$ & $6.13^{\mathrm{a}} \pm 0.98$ & $6.76^{\mathrm{a}} \pm 0.42$ & 6.29 \\
\hline $50 \%$ & & $6.28 \mathrm{a} \pm 0.31$ & $5.86^{\mathrm{a}} \pm 0.75$ & $5.51^{\mathrm{a}} \pm 1.07$ & $5.18^{\mathrm{a}} \pm 0.96$ & $5.70^{\mathrm{a}} \pm 0.77$ & 5.69 \\
\hline $75 \%$ & & $6.12^{\mathrm{a}} \pm 0.30$ & $6.13^{\mathrm{a}} \pm 0.38$ & $5.48^{\mathrm{a}} \pm 0.41$ & $5.18 \mathrm{a} \pm 0.96$ & $5.29^{\mathrm{b}} \pm 0.48$ & 5.7 \\
\hline Mean & & 6.1 & 5.98 & 5.79 & 5.62 & 5.61 & 5.91 \\
\hline$L S D(0.05)$ & & 1.1 & 0.67 & 1.32 & 1.15 & 1.07 & \\
\hline$C V(\%)$ & & 9.02 & 5.61 & 11.42 & 1.07 & 8.86 & \\
\hline $0 \%$ & Tea masala & $6.20^{\mathrm{a}} \pm 0.61$ & $5.69^{\mathrm{b}} \pm 0.55$ & $6.38^{\mathrm{a}} \pm 0.37$ & $6.04^{\mathrm{ab}} \pm 0.67$ & $7.35^{\mathrm{a}} \pm 0.32$ & 6.33 \\
\hline $25 \%$ & & $6.64^{\mathrm{a}} \pm 0.75$ & $6.76^{\mathrm{a}} \pm 0.61$ & $5.85^{\mathrm{ab}} \pm 0.77$ & $6.34^{\mathrm{a}} \pm 0.37$ & $6.44^{\mathrm{ab}} \pm 0.27$ & 6.41 \\
\hline $50 \%$ & & $6.44^{\mathrm{a}} \pm 0.54$ & $6.46^{\mathrm{ab}} \pm 0.31$ & $5.72^{\mathrm{ab}} \pm 0.23$ & $5.32^{\mathrm{b}} \pm 0.25$ & $6.37^{\mathrm{b}} \pm 0.41$ & 6.06 \\
\hline $75 \%$ & & $6.27^{\mathrm{a}} \pm 1.10$ & $6.69^{\mathrm{a}} \pm 0.73$ & $5.06^{\mathrm{b}} \pm 0.75$ & $5.16^{\mathrm{b}} \pm 0.45$ & $5.61^{\mathrm{b}} \pm 0.70$ & 5.76 \\
\hline Mean & & 6.39 & 6.4 & 5.75 & 5.72 & 6.44 & 6.14 \\
\hline$L S D(0.05)$ & & 1.46 & 0.89 & 1.09 & 0.91 & 0.91 & \\
\hline$C V(\%)$ & & 11.45 & 6.96 & 9.46 & 7.92 & 7.11 & \\
\hline
\end{tabular}

Values are Means $\pm S D$ of 30 replicates. Means in the same column with the same letter(s) are not significantly diffrent $(P>0.05)$. 
Table 2(b). Sensory evaluation of black tea (TRFK 6/8) with Cinnamon, nutmeg and ginger spices at less than 25\% Spice $(\mathrm{w} / \mathrm{w})$

\begin{tabular}{|c|c|c|c|c|c|c|c|}
\hline Concentration & Spice & Taste & Smell & Texture & Acceptability & Colour & Mean \\
\hline $0 \%$ & Cinnamon & $5.07^{b} \pm 0.28$ & $4.23^{b} \pm 0.75$ & $5.77^{b} \pm 0.25$ & $5.03^{b} \pm 0.49$ & $5.18^{b} \pm 0.94$ & 5.06 \\
\hline $5 \%$ & & $6.55^{a} \pm 0.40$ & $6.28^{a b} \pm 1.60$ & $7.18^{a} \pm 0.65$ & $7.10^{a} \pm 1.49$ & $6.30^{a b} \pm 0.93$ & 6.68 \\
\hline $10 \%$ & & $6.73^{a} \pm 0.88$ & $5.60^{a b} \pm 1.43$ & $7.08^{a} \pm 0.88$ & $7.17^{a} \pm 0.76$ & $7.13^{a} \pm 0.34$ & 6.74 \\
\hline $15 \%$ & & $6.33^{a} \pm 0.58$ & $5.12^{a b} \pm 0.77$ & $6.97^{a} \pm 0.45$ & $5.82^{b} \pm 0.35$ & $6.15^{a b} \pm 0.63$ & 6.08 \\
\hline $25 \%$ & & $6.90^{a} \pm 0.66$ & $6.75^{a} \pm 1.39$ & $6.27^{a b} \pm 0.48$ & $6.80^{a} \pm 0.58$ & $6.33^{a b} \pm 1.38$ & 6.61 \\
\hline Mean & & 6.32 & 5.6 & 6.65 & 6.38 & 6.22 & 6.23 \\
\hline$L S D(0.05)$ & & 1.08 & 2.27 & 1.08 & 0.91 & 1.16 & \\
\hline$C V(\%)$ & & 9.09 & 21.56 & 8.65 & 7.57 & 9.99 & \\
\hline $0 \%$ & Lemon grass & $6.13^{a b} \pm 1.01$ & $5.82^{a} \pm 1.01$ & $5.95^{a} \pm 0.59$ & $6.18^{a b} \pm 1.29$ & $6.24^{a b} \pm 0.81$ & 6.06 \\
\hline $5 \%$ & & $5.69^{b} \pm 1.00$ & $6.34^{a} \pm 0.56$ & $6.73^{a} \pm 1.10$ & $6.46^{a b} \pm 0.93$ & $6.54^{a} \pm 0.64$ & 6.35 \\
\hline $10 \%$ & & $6.47^{a} \pm 0.50$ & $6.01^{a} \pm 0.81$ & $6.31^{a} \pm 0.27$ & $6.74^{a} \pm 0.66$ & $6.01^{b c} \pm 0.51$ & 6.31 \\
\hline $15 \%$ & & $6.51^{a} \pm 0.65$ & $6.24^{a} \pm 0.97$ & $6.08^{a} \pm 0.56$ & $5.63^{a b} \pm 0.58$ & $5.79^{d c} \pm 0.56$ & 6.05 \\
\hline $25 \%$ & & $5.59^{b} \pm 0.90$ & $5.91^{a} \pm 1.78$ & $5.47^{a} \pm 0.91$ & $5.07^{b} \pm 0.68$ & $5.55^{d} \pm 0.57$ & 5.52 \\
\hline Mean & & 6.08 & 6.06 & 6.11 & 6.02 & 6.03 & 6.06 \\
\hline$L S D(0.05)$ & & 0.75 & 1.31 & 1.31 & 1.48 & 0.4 & \\
\hline$C V(\%)$ & & 6.53 & 11.49 & 11.4 & 13.04 & 3.55 & \\
\hline $0 \%$ & Ginger & $5.36^{b} \pm 0.34$ & $4.44^{b} \pm 0.96$ & $4.71^{b} \pm 0.96$ & $4.55^{b} \pm 0.39$ & $5.60^{b} \pm 0.85$ & 4.93 \\
\hline $5 \%$ & & $7.05^{a} \pm 1.19$ & $6.44^{a} \pm 1.90$ & $6.44^{a} \pm 1.50$ & $6.24^{a} \pm 1.17$ & $6.64^{a} \pm 1.43$ & 6.56 \\
\hline $10 \%$ & & $6.56^{a b} \pm 0.51$ & $5.67^{a b} \pm 0.34$ & $6.09^{a} \pm 1.47$ & $5.58^{a b} \pm 0.15$ & $6.51^{a b} \pm 0.72$ & 6.08 \\
\hline $15 \%$ & & $7.20^{a} \pm 1.06$ & $6.17^{a b} \pm 0.95$ & $5.91^{a b} \pm 1.54$ & $6.22^{a} \pm 0.84$ & $7.42^{a} \pm 0.37$ & 6.58 \\
\hline $25 \%$ & & $6.98^{a} \pm 0.60$ & $6.42^{a} \pm 0.73$ & $6.27^{a} \pm 0.46$ & $6.44^{a} \pm 0.96$ & $6.78^{a} \pm 0.39$ & 6.58 \\
\hline Mean & & 6.63 & 5.83 & 5.88 & 5.81 & 6.59 & 6.15 \\
\hline$L S D(0.05)$ & & 1.28 & 1.85 & 1.36 & 1.42 & 0.99 & \\
\hline$C V(\%)$ & & 10.24 & 16.87 & 12.28 & 12.97 & 8 & \\
\hline
\end{tabular}

Means in the same row with the same letter(s) are not significantly $(P>0.05)$ different.

The initial sensory trials were based on high quantities of spices to tea ratio ranging from $25 \%$ to $75 \%$ spice as shown in Table 2 (a). The overall mean liking was shown to be at spice to tea ratio of $25 \%$ spice for four types of spices; ginger spiced tea at 6.67, nutmeg 6.12, rosemary 6.29 and tea masala $6.41 .66 \%$ of panelists had overall liking at $25 \%$ spice to tea ratio. After establishing consumer acceptable spice to tea ratio of $25 \%$ spice, further sensory evaluation was done with low level spice to tea ratio of $0 \%, 5 \%, 10 \%, 15 \%$ and $25 \%$ spice as shown in Table 2 (b), using three of the spices ginger, lemon grass and cinnamon. Results show that generally there was no significant difference in spice to tea ratio of $5 \%, 10 \%$ and $15 \%$ for all the attributes. With an overall liking of 6 (like slightly) on a hedonic scale. 


\subsection{Costing}

Table 3(a). Prices of Spices, black tea and Spiced tea

\begin{tabular}{llll}
\hline Product & Quantity(g) & Cost of product (Kshs) & Unit Cost (Ksh/g) \\
\hline Black Tea & 200 & 165.00 & 0.82 \\
Black Tea & 200 & 180.00 & 0.90 \\
Black Tea & 200 & 200.00 & 1.00 \\
Cinnamon Spice & 100 & 77.00 & 0.77 \\
Cinnamon Spice & 100 & 79.00 & 0.79 \\
Cinnamon Spice & 50 & 37.00 & 0.74 \\
Ginger Spice & 100 & 85.00 & 0.85 \\
Ginger Spice & 100 & 82.00 & 0.82 \\
Ginger Spice & 100 & 84.00 & 0.84 \\
Ginger (black) tea & 200 & 220.00 & 1.10 \\
Ginger (black) tea & 200 & 225.00 & 1.13 \\
Ginger (black) tea & 200 & 230.00 & 1.15 \\
Masala (black) tea & 50 & 115.00 & 2.30 \\
Masala (black) tea & 50 & 125.00 & 2.50 \\
Masala (black) tea & 50 & 130.00 & 2.60 \\
Lemon grass spice & 100 & 82.00 & 0.82 \\
Lemon grass spice & 100 & 85.00 & 0.85 \\
Lemon grass spice & 100 & 86.00 & 0.86 \\
\hline
\end{tabular}

Unit Cost was calculated by dividing the product cost and quantity. Cost of commodities are in Kenya Shillings (Ksh) and that of quantity in grams $(\mathrm{g})$ prices are according to commonly available products in the year 2013.

Values of unit cost of Table 3(a) were used to generate the cost of different tea spice percentages in Table 3(b) which depicts possible costs of the cinnamon tea product at different percentage mixes.

Table 3(b). Summary of the price of black tea at different Cinnamon Spice ratios

\begin{tabular}{lllllll}
\hline Spice & Tea & Spice unit $(1 \mathrm{~g})$ & Tea unit $(1 \mathrm{~g})$ & Spice Cost/pdt & Tea Cost/pdt & Product Cost \\
\hline 0 & 100 & $0.77^{\mathrm{a}} \pm 0.03$ & $0.91^{\mathrm{a}} \pm 0.02$ & $0.00^{\mathrm{f}} \pm 0.00$ & $91.91^{\mathrm{a}} \pm 2.00$ & $91.00^{\mathrm{d}} \pm 2.00$ \\
5 & 95 & $0.77^{\mathrm{a}} \pm 0.03$ & $0.91^{\mathrm{a}} \pm 0.02$ & $3.85^{\mathrm{e}} \pm 0.13$ & $86.45^{\mathrm{b}} \pm 1.90$ & $90.30^{\text {cd }} \pm 1.83$ \\
10 & 90 & $0.77^{\mathrm{a}} \pm 0.03$ & $0.91^{\mathrm{a}} \pm 0.02$ & $7.70^{\mathrm{d}} \pm 0.25$ & $81.90^{\mathrm{c}} \pm 1.80$ & $89.60^{\text {cd }} \pm 1.66$ \\
15 & 85 & $0.77^{\mathrm{a}} \pm 0.03$ & $0.91^{\mathrm{a}} \pm 0.02$ & $11.55^{\mathrm{c}} \pm 0.38$ & $77.35^{\mathrm{d}} \pm 1.70$ & $88.90^{\mathrm{bc}} \pm 1.51$ \\
25 & 75 & $0.77^{\mathrm{a}} \pm 0.03$ & $0.91^{\mathrm{a}} \pm 0.02$ & $19.25^{\mathrm{b}} \pm 0.63$ & $68.25^{\mathrm{e}} \pm 1.50$ & $87.50^{\mathrm{b}} \pm 1.23$ \\
100 & 0 & $0.77^{\mathrm{a}} \pm 0.03$ & $0.91^{\mathrm{a}} \pm 0.02$ & $77.00^{\mathrm{a}} \pm 2.52$ & $0.00^{\mathrm{f}} \pm 0.00$ & $77.00^{\mathrm{a}} \pm 2.52$ \\
Mean & & 0.77 & 0.56 & 19.81 & 41.53 & 61.34 \\
LSD(0.05) & $<0.01$ & $<0.01$ & 1.71 & 1.36 & 2.75 \\
CV (\%) & $<0.01$ & $<0.01$ & 4.74 & 1.80 & 2.46 \\
\hline
\end{tabular}

Means in the same column with the same letter(s) are not significantly (P $\leq 0.05)$ different. Pdt $=1$ unit of product comprising of spice and tea mixed at a specified ratio 
Table 3(b) shows that there is a significant $(\mathrm{P} \leq 0.05)$ decrease in the cost of production with an increase in the quantity of spice added. But the quantity of the spice added is dependent on the consumer preference threshold value of the spices as perceived by the consumers. Sensory evaluation data and economic analysis of the spice and tea mixes have to be used in the development of spiced tea mixes which are consumer acceptable and producer profitable.

\section{Conclusion and Recommendations}

The results reported in this article have shown that preference for black tea is enhanced by blending tea with spices, although some spices are required in higher amounts than others for optimum flavoring of the spice-tea blends. The antioxidant activity of spiced tea products was down regulated by spices but preference was enhanced. Antioxidant activity of pure spices was $46.99,55.70,69.23,89.47$ and 89.89 for nutmeg, tea masala, ginger, rosemary and cinnamon respectively. Antioxidant activity has been shown to correlate with total polyphenols content in tea. these polyphenols are increasingly being gaining popularity as having potential health benefits (Pandey \& Rizvi).

Spice concentration had a great impact on the panelist rating as shown by the three best rated spices. At high concentration of $25 \% \mathrm{w} / \mathrm{w}$, lemongrass spiced tea had the highest overall mean liking followed by ginger spiced tea and cinnamon spiced tea, while at low concentration cinnamon spiced tea had the highest overall liking followed by ginger spiced tea and lemongrass spiced tea respectively. Overall sensory rating of spiced tea varied among spiced tea, nutmeg spiced tea had the lowest mean liking (5.9), while lemon grass spiced tea was rated highest (6.83).

Tea blending at 1:4 ratio of spice to tea is highly recommended, since the production cost is low and it can meet consumer demand (acceptability). Blending of tea with spices should be carefully done for individual spices, since some spices may be required at lower concentrations while others at a higher concentration. High antioxidant spices may also cause potential health problems as demonstrated by Skulas-Ray et al. (2011).

Pricing of the spiced tea blends differed with the type of tea and amount of added spice. From the three best rated spices, cinnamon spiced tea was the least expensive followed by ginger spiced tea and lemon grass spiced tea. Therefore, this study recommends the best spice as cinnamon since it exhibited high antioxidant activity and the highest overall mean liking. Least amounts of this spice were also used to achieve desired taste, smell, texture, acceptability, and color. Nutmeg spice was rated poorly and to get a similar effect as cinnamon, large amounts of the spice were required which further down regulated the antioxidant activity of tea significantly and increased cost of production. This study leaves a lot of room for research on preference studies between spiced tea and other beverages.

\section{Acknowledgments}

The authors acknowledge financial support from the National Commission for Science, Technology and innovation (NACOSTI), Kenya and the Kenya Agricultural and Livestock Research Organization-Tea Research Institute (KALRO-TRI). Reviewers and technical support staff of KALRO-TRI are also acknowledged.

\section{References}

Anon. (2011). Tea Board of Kenya Statistics. Ngong road, Nairobi.

Benkeblia, N. (2004). Antimicrobial activity of essential oil extracts of various onions (Allium cepa) and garlic (Allium sativum). LWT - Food Science and Technology, 37(2), 263-268. http://doi.org/10.1016/j.lwt.2003.09.001

Bullerman, L. B., Lieu, F. Y., \& Seier, S. A. (1977). Inhibition of growth and aflatoxin production by cinnamon and clove oils. Cinnamic aldehyde and eugenol. Journal of Food Science, 42(4), 1107-1109. http://doi.org/10.1111/j.1365-2621.1977.tb12677.x

Carpenter, R. G. (1960). Principles and procedures of statistics, with special reference to the biological sciences. The Eugenics Review, 52(3), 172-173. http://doi.org/10.1002/bimj.19620040313

Cass, T. (1980). Statistical Methods in Management: Book 2. Retrieved from

Choi, C. W., Kim, S. C. S. K., Hwang, S. S., Choi, B. K., Ahn, H. J., Lee, M. Y., ... Kim, S. C. S. K. (2002). Antioxidant activity and free radical scavenging capacity between Korean medicinal plants and flavonoids by assay-guided comparison. Plant Science, 163(6), 1161-1168. http://doi.org/10.1016/S0168-9452(02)00332-1.

Cochran, W. C. (1977). Snedecor G W \& Cochran W G. Statistical methods applied to experiments in agriculture 
and biology. 5th ed. Ames, Iowa: Iowa State University Press, 1956. Citation Classics, 19, 1.

Crespy, V., \& Williamson, G. (2004). A review of the health effects of green tea catechins in in vivo animal models. The Journal of Nutrition, 134(12 Suppl), 3431S-3440S.

Crozier, A., Jaganath, I., \& Clifford, M. (2009). Dietary phenolics: chemistry, bioavailability and effects on health. Natural Product Reports.

Guynot, M. E., Ramos, A. J., Setó, L., Purroy, P., Sanchis, V., Marín, S., ... Marin, S. (2003). Antifungal activity of volatile compounds generated by essential oils against fungi commonly causing deterioration of bakery products. Journal of Applied Microbiology, 94(5), 893-9.

Herath, H., \& De Silva, S. (2011). Strategies for Competitive Advantage in Value Added Tea Marketing. Tropical Agricultural Research, 22(3), 251-262.

Holt, P. R., Katz, S., \& Kirshoff, R. (2005). Curcumin therapy in inflammatory bowel disease: a pilot study. Digestive Diseases and Sciences, 50(11), 2191-2193. http://doi.org/10.1007/s10620-005-3032-8

IDF. (1987). Sensory evaluation of dairy product. In In I.D. Federation, FIL-IDF Standard 99A. Bruxelles: Belgium: International Dairy Federation.

ISO14502-2-2005E. (2005). Determination of substances characteristic of green and black tea-Part 2. Contents of catechins in green tea; method using High Performance Liquid Chromatography. In International Standards Organizations Analytical Methods. ISO.

ITC. (2010). International Tea Committee- Annual Bulletin of Statistics London.

Jagetia, G. C., Baliga, M. S., Venkatesh, P., \& Ulloor, J. N. (2003). Influence of ginger rhizome (Zingiber officinale Rosc) on survival, glutathione and lipid peroxidation in mice after whole-body exposure to gamma radiation. Radiation Research, 160(5), 584-92.

Karori, S. M., Wachira, F. N., Ngure, R. M., \& Mireji, P. O. (2014). Polyphenolic composition and antioxidant activity of Kenyan Tea cultivars. Journal of Pharmacognosy and Phytochemistry, 3(4), 105-116.

Karori, S. M., Wachira, F. N., Wanyoko, J. K., \& Ngure, R. M. (2007). Antioxidant capacity of different types of tea products. African Journal of Biotechnology, 6(19).

Kerio, L. C., Wachira, F. N., Wanyoko, J. K., \& Rotich, M. K. (2013). Total polyphenols, catechin profiles and antioxidant activity of tea products from purple leaf coloured tea cultivars. Food Chemistry, 136(3-4), 1405-13. http://doi.org/10.1016/j.foodchem.2012.09.066

Koech, K., \& Wachira, F. (2013). Antibacterial and Synergistic Activity of Different Tea Crude Extracts against Antibiotic Resistant S. Aureus, E. Coli and a Clinical Isolate of S. Typhi. Science Journal of ....

Lai, P. K., \& Roy, J. (2004). Antimicrobial and chemopreventive properties of herbs and spices. Current Medicinal Chemistry, 11(11), 1451-60.

Lelgo, S. K., Kamunya, S. M., Ochanda, S. O., \& Wanyoko, J. K. (2011). The effect of processing technique on biochemical composition of adapted Kenyan and introduced Japanese and Chinese Tea germplasm. Tea, $32(1), 22-23$.

Meilgaard, M., Civille, G., \& Carr, B. (1999). Sensory evaluation techniques (3rd Ed.). Boca Raton, F1: CRC Press.

Miller, I. J., \& Bartoshuk, L. M. (1991). Taste perception, taste bud distribution, and spatial relatioships. Smell and Taste in Health and Disease, (11), 205-233.

Mohd Nasir, N. (2012, April 18). Determination of tea polyphenols, Thearubigins (TR), Theaflavins (TR) and Teabrownins (TB) in tea bag and process tea leaves in Malaysian market / Noraslina Mohd Nasir.

Moraes, M. (1993). In Me 'todos para avaliac, a o sensorial de alimentos, 8th Edition. Unicamp, Campinas, Brazil.

Nielsen, P. V, \& Rios, R. (2000). Inhibition of fungal growth on bread by volatile components from spices and herbs, and the possible application in active packaging, with special emphasis on mustard essential oil. International Journal of Food Microbiology, 60(2-3), 219-29.

Ochanda, S. O., Wanyoko, J. K., Kamau, D. M., Kamunya, S. M., Faraj, A. K., \& Onyango, C. (2011). The effect of selected spices on un-aerated tea product from clone TRFK 303/57. Tea Journal, 32(2), 88-99.

Ochanda, S. O., Wanyoko, J. K., \& Ruto, H. K. (2015). Effect of Spices on Consumer Acceptability of Purple 
Tea. Food and Nutrition Sciences, 06(08), 703-711. http://doi.org/10.4236/fns.2015.68073

Pandey, K. B., \& Rizvi, S. I. Plant polyphenols as dietary antioxidants in human health and disease. Oxidative Medicine and Cellular Longevity, 2(5), 270-8. http://doi.org/10.4161/oxim.2.5.9498

Roberts, E. A. H., \& Smith, R. F. (1961). Spectrophotometric measurements of theaflavins and thearubigins in black tea liquors in assessments of quality in teas. Analyst, 86(1019), 94-98.

Ruto, H. K., Moseti, K. O., Koech, R. K., \& Wanyoko, J. K. (2014). Methods developed for the analysis of major biochemical compounds found in tea: A review. In 1st International Confrence Maasai Mara University, 11-14 November. Maasai Mara University.

SAS Institute Inc. (n.d.). SAS 9.1.3 Help and Documentation, Cary, NC: SAS Institute Inc.

Skulas-Ray, A. C., Kris-Etherton, P. M., Teeter, D. L., Chen, C. Y. O., Heuvel, J. P. Vanden, \& West, S. G. (2011). A high antioxidant spice blend attenuates postprandial insulin and triglyceride responses and increases some plasma measures of antioxidant activity in healthy, overweight men. The Journal of Nutrition, 141(8), $1451-1457$.

Wang, H., Provan, G. J., \& Helliwell, K. (2000). Tea flavonoids: their functions, utilisation and analysis. Trends in Food Science \& Technology, 11(4), 152-160.

Willson, K. C., \& Clifford, M. N. (1992). Tea: cultivation to consumption.

Yen, G. C., \& Duh, P. Der. (1994). Scavenging Effect of Methanolic Extracts of Peanut Hulls on Free-Radical and Active-Oxygen Species. Journal of Agricultural and Food Chemistry, 42(3), 629-632. http://doi.org/10.1021/jf00039a005

Zong-Mao, C., Wang, H.-F., Xiao-Qingyou, \& Xu, N. (2005). In Tea bioactivity and therapeutic potential. In Z. Yong-su (Ed.), Tea (p. 78). London and New York: Taylor and Francis e- library.

\section{Copyrights}

Copyright for this article is retained by the author(s), with first publication rights granted to the journal.

This is an open-access article distributed under the terms and conditions of the Creative Commons Attribution license (http://creativecommons.org/licenses/by/3.0/). 